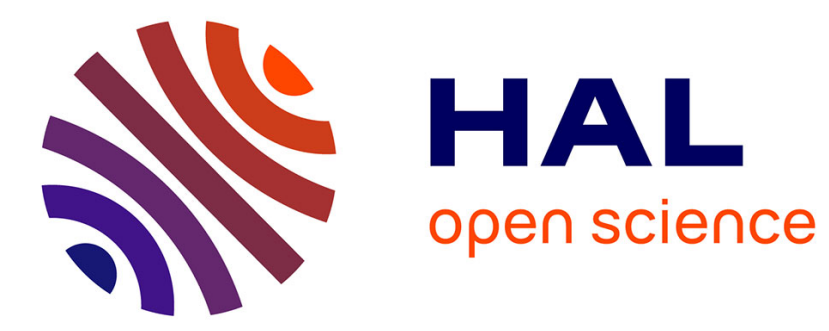

\title{
Triggering granular avalanches with ultrasound
}

\author{
J. Léopoldès, X. Jia, A. Tourin, A. Mangeney
}

\section{To cite this version:}

J. Léopoldès, X. Jia, A. Tourin, A. Mangeney. Triggering granular avalanches with ultrasound. Physical Review E , 2020, 102, p.1535-1581. 10.1103/PhysRevE.102.042901 . insu-03584466

\section{HAL Id: insu-03584466 https://hal-insu.archives-ouvertes.fr/insu-03584466}

Submitted on 22 Feb 2022

HAL is a multi-disciplinary open access archive for the deposit and dissemination of scientific research documents, whether they are published or not. The documents may come from teaching and research institutions in France or abroad, or from public or private research centers.
L'archive ouverte pluridisciplinaire HAL, est destinée au dépôt et à la diffusion de documents scientifiques de niveau recherche, publiés ou non, émanant des établissements d'enseignement et de recherche français ou étrangers, des laboratoires publics ou privés. 


\title{
Triggering granular avalanches with ultrasound
}

\author{
J. Léopoldès $\odot,{ }^{1,2,{ }^{*}}$ X. Jia $\odot,{ }^{1,2, \dagger}$ A. Tourin, ${ }^{1}$ and A. Mangeney ${ }^{3}$ \\ ${ }^{1}$ Institut Langevin, ESPCI Paris, Université PSL, CNRS, 75005 Paris, France \\ ${ }^{2}$ Université Gustave Eiffel, 75454 Marne-la-Vallée Cedex 2, France \\ ${ }^{3}$ Institut de Physique du Globe de Paris, Université de Paris, 75005 Paris, France
}

(Received 30 June 2020; accepted 11 September 2020; published 6 October 2020)

\begin{abstract}
Granular flows triggered by vibration below the avalanche angle are ubiquitous in nature. However, the mechanism of triggering and the nature of the resulting flow are not fully understood. Here we investigate the triggering of the shear instability of granular layers by nanometer-amplitude ultrasound close to the static threshold. We find that such small-amplitude and high-frequency sound waves provoke unjamming, resulting in a self-accelerated inertial flow or a creeplike regime which stops flowing after the removal of ultrasound. We show that these effects are due to the reduction of interparticle friction at grain contacts by the shear acoustic lubrication. Our observations are consistent with the bistability inherent to velocity-weakening friction models [e.g., Jaeger et al., Europhys. Lett. 11, 619 (1990)]. This work should help to understand the local and remote triggering of landslides and earthquakes by seismic waves.
\end{abstract}

DOI: 10.1103/PhysRevE.102.042901

\section{INTRODUCTION}

Laboratory studies of the frictional instability in sheared granular media have emerged as a powerful tool for investigating dynamics of seismic faults, landslides, and avalanches [Figs. 1(a) and 1(b)] [1,2]. An important and challenging issue in seismic hazard is to understand the remote dynamics triggering of earthquake by impinging seismic (elastic) waves at microstrain amplitude [3]. Moreover, recent observations showed that perturbations from local foreshocks activities are probably a part of the earthquake nucleation process $[2,4]$ and that large rockfall events and avalanches can be triggered by volcanic seismicity [5]. Indeed, dynamic stress from seismic waves can perturb fault systems that are close to the yield stress, e.g., due to tectonic stresses $\tau \lesssim \tau_{s}$ [Fig. 1(c)], and force failure earlier in time relative to an unperturbed fault or cliff. Understanding the mechanics of local and remote triggering of landslides will go a long way in quantifying seismic hazard.

Advances in granular physics and acoustics have paved the way for understanding how and under what conditions impinging seismic waves may trigger a fault slip. A granular medium is an assembly of discrete macroscopic solid grains that interact with each other by dissipative contact forces. Unlike ordinary solids and liquids, static and dynamic properties of dense granular media are determined by inhomogeneous contact force networks, exhibiting multiple metastable configurations. Sound waves propagating from grain to grain provide not only a unique probe of such networks (often opaque) [6-9] but also a controlled perturbation via elastic softening and frictional dissipation [10-14]. Granular media

\footnotetext{
*julien.leopoldes@univ-mlv.fr

†xiaoping.jia@espci.fr
}

undergo a transition from a jammed solid state to a flowing liquid state when the external shear exceeds the static yield stress [15].

Theoretical and experimental studies suggest that this transition is a subcritical bifurcation [16-19] [sketched in Fig. 1(d)], with dynamics similar to solid friction at multicontact interfaces [20] [insets of Figs. 1(a) and 1(b)] well-described by the rate and state constitutive law by Dieterich, Rice, and Ruina [1,2]. Burridge and Knopoff have also used this kind of velocity-weakening friction in their spring-block model to describe the earthquake stick-slip dynamics $[21,22]$. Here, we define $\mu=\tau / \sigma_{n}=F_{t} / F_{n}$ the shear stress (force) normalized by the normal stress (force) from which the static and dynamic coefficients of friction $\mu_{s, d}=$ $\tau_{s, d} / \sigma_{n=} F_{s, d} / F_{n}$ follow. Here $\tau_{s}$ and $F_{s}$ are the static friction stress and force at yield while $\tau_{d}$ and $F_{d}$ are the dynamic friction stress and force. In the inclined plane geometry of angle $\theta$ such as in the present study [Fig 1(b)], we have $\mu=\tan \theta$ and $\mu_{s}=\tan \theta_{\mathrm{m}}$, with $\theta_{m}$ the (maximum) angle of avalanche. The angle of repose $\theta_{\mathrm{r}}$ being a few percent lower than $\theta_{m}$ [23-26] corresponds to the dynamic friction $\mu_{d}=\tan \theta_{\mathrm{r}}$ at the minimum shear load [Fig. 1(d)].

Previous works $[20,27-29]$ showed that for shear forces far below the threshold $\mu \ll \mu_{s}$, both granular layers and rough solid interfaces [Figs. 1(a), 1(b), and insets] respond elastically as shown in Fig. 1(c), via the reversible deformation of contacting grains or/and asperities, in the jammed state (region I). For $\mu \lesssim \mu_{s}$, nonlinear response occurs with creeplike irreversible sliding. For $\mu \gtrsim \mu_{s}$, the system yields and starts to slide over a transient characteristic distance $D_{c}$ (slip weakening) $[1,2,20]$ before reaching the steady-flow region II/III at a velocity $V$ or shear rate $\dot{\gamma}$ imposed by the load (to be described in the following). In the representation $\mu=\mu(V)$ or $\mu(\dot{\gamma})$ plotted in Fig. 1(d), the velocity weakening region $\left(\dot{\gamma}<\dot{\gamma}_{0}\right)$, referred to as "II," is unstable and can lead to intermittent 
(a)

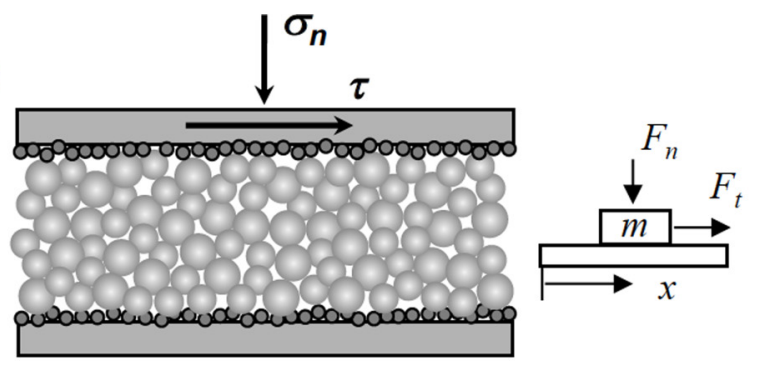

(b)

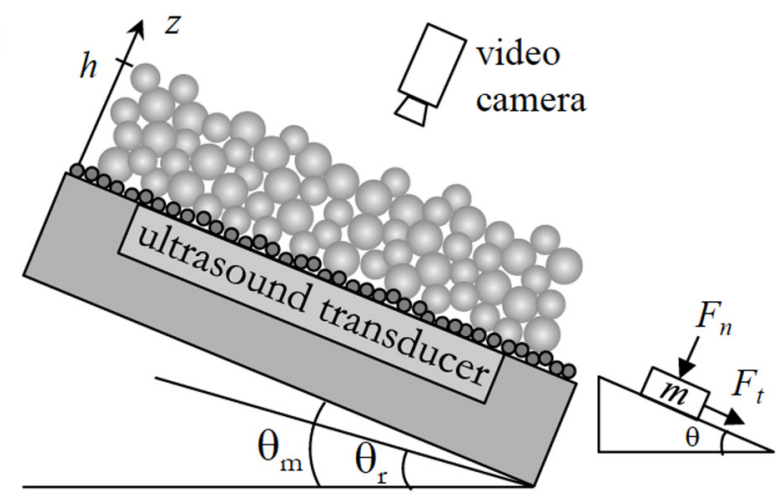

(c)

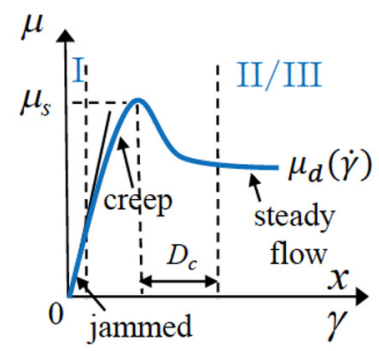

(d)

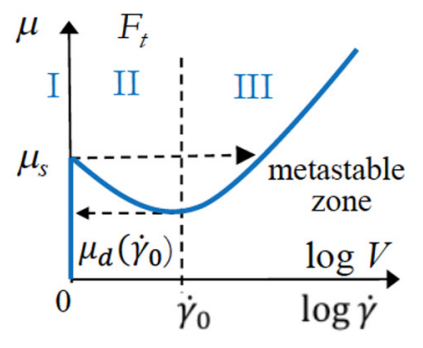

FIG. 1. (a) Schematic cross section of a granular layer under shear (e.g., fault gouge). (b) Flow of a granular layer on an inclined plane at angle $\theta$. The angle of avalanche is $\theta_{m}$ and the angle of repose is $\theta_{r}$. (c) Typical plot of the normalized shear stress (force) $\mu=\tau / \sigma_{n}=F_{t} / F_{n}$ vs strain $\gamma$ or displacement $x$. Region I corresponds to the jammed state with a linear elastic response. It is followed by slow creep motion when approaching the threshold $\mu_{s}$. After yielding, the system slides over a characteristic distance $D_{c}$ before reaching the steady flow at a velocity or rate imposed by shear load; as shown in (d) it can be stable (III) or unstable (II). (d) Typical plot of $\mu$ vs flow rate $\dot{\gamma}$ or velocity $V$ with the different states as in (c). Region I appearing as a vertical line corresponds to the jammed state, region II $\left(\dot{\gamma}<\dot{\gamma}_{0}\right)$ to the unstable intermittent flow/slip, and region III $\left(\dot{\gamma}>\dot{\gamma}_{0}\right)$ to the stable inertial flow/slip where $\dot{\gamma}_{0}$ is the shear rate corresponding to the minimum friction.

flow behavior (e.g., stick-slip). Such frictional instability results from an aging-rejuvenation competition acting within the micrometer-sized contacting asperities [20], which can also be modeled by the micro- or nanoblocks coupled elastically by springs [30]. For high flow rates $\left(\dot{\gamma}>\dot{\gamma}_{0}\right)$, the slope of $\mu(\dot{\gamma})$ is positive and ensures the stability of the steady flow as in region III.

The possible failure of a granular medium, such as the sandpile, caused by external vibrations, has been known for a long time in engineering and geophysical applications, however a unified physical description still lacks. The vibrations considered are most of the time of large-amplitude $U_{0} \gtrsim d$ with $d$ the grain size and low-frequency $f<f_{0}$. where $f_{0}$ is a characteristic frequency determined by the stiffness of Hertzian contacts $[20,28]$. The amount of shaking is usually estimated by the reduced peak acceleration of the grain $\Gamma=a / g=(2 \pi f)^{2} U_{0} / g$ with $g$ the gravity. When $\Gamma>1$, vertical vibrations cancel almost normal forces exerting on the grain (confined under gravity) and modify consequently the spatial arrangement of grains, resulting in phenomena such as compaction, convection, shear banding, to mention a few [31-33]. This is similar to the oscillation effect on the normal stress facilitating sliding $[34,35]$ and also to the scenario of the acoustic fluidization in a confined continuous medium. In this scenario, the acoustic pressure $p_{a}=(\rho c) v_{\mathrm{a}}$, with $c$ the sound speed and $v_{a}$ the vibration velocity, is expected to temporally relieve the pressure of the overburden, thereby decreasing the yield stress [36]. For horizontal vibrations, whenever $\Gamma>\mu$, the grains in the top layers of free surface slide against each other [37-39] akin to the slider on an inclined frictional plane $[28,29]$. Other studies claim that shaking should be parametrized by the vibration velocity $v_{\mathrm{a}}=(2 \pi f) U_{0}$ squared, because the vibrations interact with the medium in terms of a granular temperature $T_{g}$ proportional to the kinetic energy of the grain $T_{g} \sim(1 / 2) m v_{\mathrm{a}}^{2}$ with $m$ the grain mass [33]. More recent works suggest rather a collisionlike pressure term, $p_{c} \sim$ $(1 / 2) \rho v_{\mathrm{a}}^{2}$ with $\rho$ the granular mass density $[40,41]$.

However, the above scenarios, involving large-amplitude vibrations, cannot explain the dynamic earthquake triggering by seismic waves at micro- and nanostrain amplitude [2,3], nor the laboratory experiments using nanometer-amplitude ultrasound to soften the material modulus by $30 \%$ via nonlinear dynamics $[10,12]$. Also, some modifications of the stick-slip cycle by ultrasound remain unexplained [11]. In these situations, the oscillation frequency of ultrasound $f \geqslant 40 \mathrm{kHz}$ is high compared to the characteristic frequency $f_{0} \sim 5 \mathrm{kHz}$ in millimeter-thick granular layers $[20,28]$ so that grains cannot have normal motion, due to inertia, in order to suppress the weight of the overburden. On the other hand, for a nanometer ultrasonic vibration, the collisionlike pressure estimated as $p_{c} \sim 10^{-4} \mathrm{~Pa} \ll \sigma_{n}(\sim 10 \mathrm{~Pa})$ is too small to be considered.

In this work, we focus on the triggering of granular instabilities by small-amplitude and high-frequency sound waves. In such conditions, we have already evidenced another mechanism in which the local threshold friction force at one grain contact can be reduced by a shear acoustic lubrication, 
TABLE I. Dependence of the avalanche angle on surface roughness and layer thickness.

\begin{tabular}{|c|c|c|c|c|c|}
\hline Roughness & Smooth & \multicolumn{3}{|c|}{ Intermediate } & Rough \\
\hline Thickness $h / d$ & 3 & 3 & 6 & 11.6 & 3 \\
\hline$\theta_{m}(d \approx 0.5 \mathrm{~mm})$ & $16.5^{\circ} \pm 1^{\circ}$ & $20.2 \pm 1^{\circ}$ & $16.5^{\circ}$ & & $36^{\circ} \pm 1^{\circ}$ \\
\hline$\theta_{m}(d \approx 0.1 \mathrm{~mm})$ & & & & $22.5^{\circ}$ & \\
\hline
\end{tabular}

lowering the effective interparticle friction coefficient [42]. However, the question is whether such local effects could give rise to collective motion since small-amplitude ultrasound does not induce grain displacement per se at the relevant length-scale (i.e., grain diameter $d$ ) during avalanches. We will show that depending on the interplay between the ultrasound amplitude and the driving force, granular layers can be found in different states: jammed solid, slow creep flow, and fast inertial liquid. Our experimental results are analyzed on the basis of velocity-weakening friction models.

\section{EXPERIMENTS}

The experimental setup is shown in Fig. 1(b). A given mass of glass beads (of diameter $d$ ) is deposited on the surface of an ultrasonic transducer by pluviation, building a flat and homogeneous granular layer of controlled thickness $h$ and packing density (solid volume fraction $\phi \sim 0.6$ ). After a waiting time of $10 \mathrm{~min}$ to overcome aging effects $[10,12]$ and ensure a controlled initial state, the avalanche angle $\theta=\theta_{m}$ is measured as a reference state by inclining the plane until a continuous homogeneous flow occurs (see video 1 in the Supplemental Material [43]), for different granular layers and surface roughnesses.

The surface of the transducer ("smooth") is used as it is, or with a controlled roughness by attaching to it, with an acoustically transparent adhesive tape, some glass beads of various diameters: $10 \mu \mathrm{m}$ for "intermediate" and $100 \mu \mathrm{m}$ for "rough" surfaces. We investigate granular layers composed of slightly polydisperse glass beads (about $20 \%$ in size): large $(d \approx 500 \mu \mathrm{m})$ and small $(d \approx 100 \mu \mathrm{m})$. As reported previously, the avalanche angle depends on the thickness of the granular layer: a thicker $h$ leads to a smaller $\theta_{m}$ [23,24,26]. The avalanche angles measured for various surface roughness and layer thickness are given in Table I.

The ultrasound vibration is delivered by transmitting a continuous sinusoidal signal to a longitudinal piezoelectric transducer at two different frequencies $f=40$ and $100 \mathrm{kHz}$. The input voltage varies from 10 to $100 \mathrm{~V}$, corresponding to vibration amplitudes ranging from $U_{0} \sim 5$ to $50 \mathrm{~nm}$ calibrated with an optical interferometer $[10,12]$. Some experiments have been conducted with shear transducers having their polarization oriented either along the slope or perpendicular to it, leading to a similar phenomenology as that reported for the longitudinal transducers.

Here, we focus on those cases where granular layers are initially jammed at an angle of inclination $\theta<\theta_{r}$. The amplitude of ultrasound is increased until the first grain motions are detected. More precisely, video of grain positions is recorded with a camera at 60 frames/s and the trajectories and accord- ingly the flows of the grains are computed with a tracking procedure [44]. The resolution of grain motions is limited by the pixel size of images that is about $5 \mu \mathrm{m}$. The Probability Distribution Function (PDF) of grain velocities is then obtained as shown in Fig. 2 where we plot both the transverse (a) and longitudinal (along the slope) components (b). The central part of the PDF is fitted with a Gaussian curve. From these PDFs, we deduce the average flow velocity $V_{\text {flow }}$ and the standard deviation $\delta V$. For the transverse component, the PDF are symmetrically distributed around $V_{\perp}=0$ as there is no preferential path in that direction. Along the longitudinal direction, the PDF are asymmetric around $V_{\|}=0$ with a nonzero average flow velocity. All distributions have large tails, due to both the heterogeneous nature of the granular flow [45] and some boundary effects due to the geometry of the transducers.

\section{RESULTS}

\section{A. Dynamics of triggered granular flows}

Figure 3(a) depicts the normalized friction $\mu / \mu_{s}(=$ $\left.\tan \theta / \tan \theta_{\mathrm{m}}\right)$ vs the dimensionless flow velocity $\tilde{V}=$ $V_{\text {flow }} /(g d)^{1 / 2}$ on the intermediate roughness surface. Depending on the distance to yielding, we observed different regimes of flow triggered by ultrasound. For $\mu / \mu_{\mathrm{s}} \ll 1$ and too small amplitudes of ultrasound, the system is in the jammed state I, defined by the vertical (plain) line $\tilde{V}=0$ as in Fig. 1(d). In region II close to region I, flow occurs at a very small averaged velocity $\tilde{V} \sim 10^{-2}$. Closer to yielding, for $\mu / \mu_{s} \sim 0.9$, we find a rather abrupt dynamic transition where the system jumps, around a characteristic $\tilde{V}^{*} \sim 3 \times 10^{-2}$ (dotted line) from an intermittent creep flow to a much faster, continuous inertial flow (dashed line). As shown in Fig 3(b), $\tilde{V}^{*}$ corresponds to a marked peak in the evolution of the transverse relative velocity fluctuations $\delta V / V_{\text {flow }}$, indicating the boundary of a phase transition to the stable flow state. Above the transition, the granular layer flows with a typical $\tilde{V}>10^{-1}$, close to the order of magnitude of the flow velocity of a natural avalanche, which is used here arbitrarily to define region III for the onset of stable flow.

The observed ultrasound-induced granular flow may be characterized by different states as provided by the order of magnitude of the typical average velocity. This is shown in Fig. 3(c) where $\mu / \mu_{s}$ is replotted from Fig. 3(a) but as a function of the ultrasound amplitude $U_{0}$. Such a phase diagram shows that transition between the jammed state, the creep flow state, and stable flow state shall occur via arbitrary paths in the $\left(\mu, U_{0}\right)$ plane. Below, we shall suggest a local friction model to describe the line schematically delimiting jammed state I and flowing state I/II.

Moreover, inspection of Fig. 3(a) suggests that in the dynamic transition zone between regions II and III, the flow velocity is an increasing function of the amplitude at imposed shear $\mu$. For example, for $\mu / \mu_{s} \approx 0.85$, when the ultrasound amplitude $U_{0}$ is increased from 15 to $45 \mathrm{~nm}$, the flow velocity is increased by $\Delta V_{\text {flow }} \approx 3 \mathrm{~mm} / \mathrm{s}$, which gives an increasing factor about $\Delta V_{\text {flow }} / \Delta U_{0} \sim 10^{5} \mathrm{~s}^{-1}$. We confirm this trend with more precision by showing additional measurements of $\tilde{V}$ from experiments achieved with a fine increase of the 

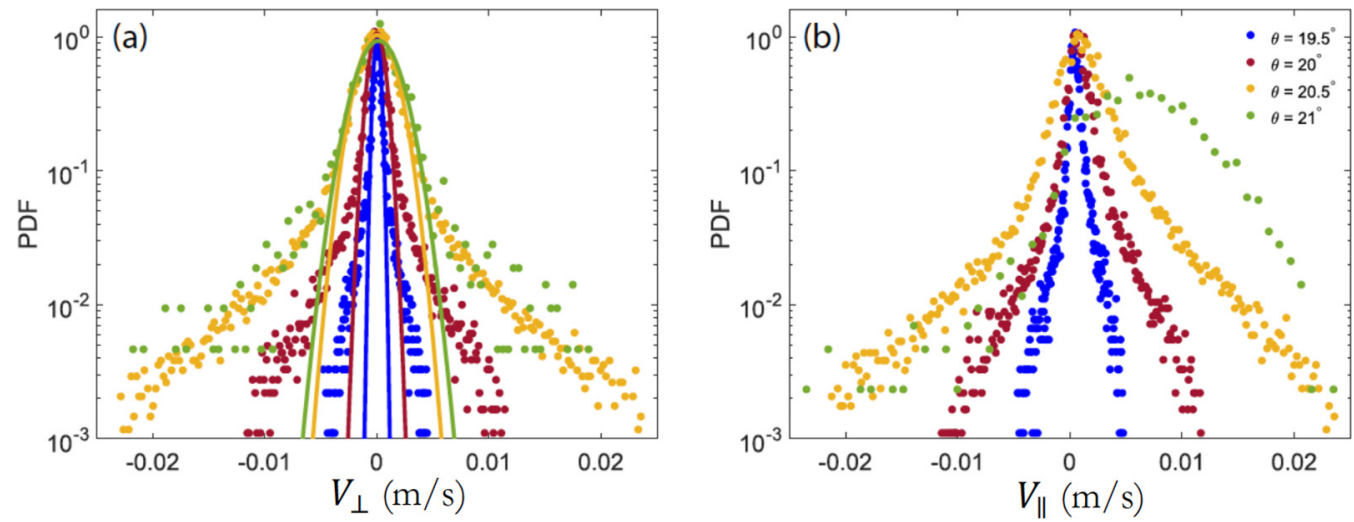

FIG. 2. PDF of grain velocities driven by ultrasound $\left(U_{0}=15 \mathrm{~nm}\right)$ in a layer of thickness $h / d=11.6(d=100 \mu \mathrm{m})$ deposited on the surface of intermediate roughness. The angle of inclination $\theta$ varies from $19.5^{\circ}$ to $21^{\circ}\left(\theta_{m} \approx 22.5^{\circ}\right)$. (a) PDF of the transverse velocity, symmetric around $V_{\perp}=0$. (b) PDF of the longitudinal velocity $V_{\|}$along the slope, from which the average flow velocity $V_{\text {flow }}$ is computed.

ultrasound amplitude at constant inclination in a layer of large beads $d=500 \mu \mathrm{m}$ as shown by Fig. 3(d). At $\mu / \mu_{s}=0.73$, the flow velocity increases continuously with amplitude $U_{0}>$ $25 \mathrm{~nm}$, such that $\Delta V_{\text {flow }} / \Delta U_{0}=1.1 \times 10^{5} \mathrm{~s}^{-1}$, a value consistent with the preceding measurement. In this region (II) where the flow velocity is controlled by ultrasound, the granular layer stops flowing when the ultrasound is removed (see video 2 in the Supplemental Material [43]).

By contrast, as shown in Fig. 3(a), when the inclination increased close to the threshold at $\mu / \mu_{s} \leqslant 1$, the dynamics of
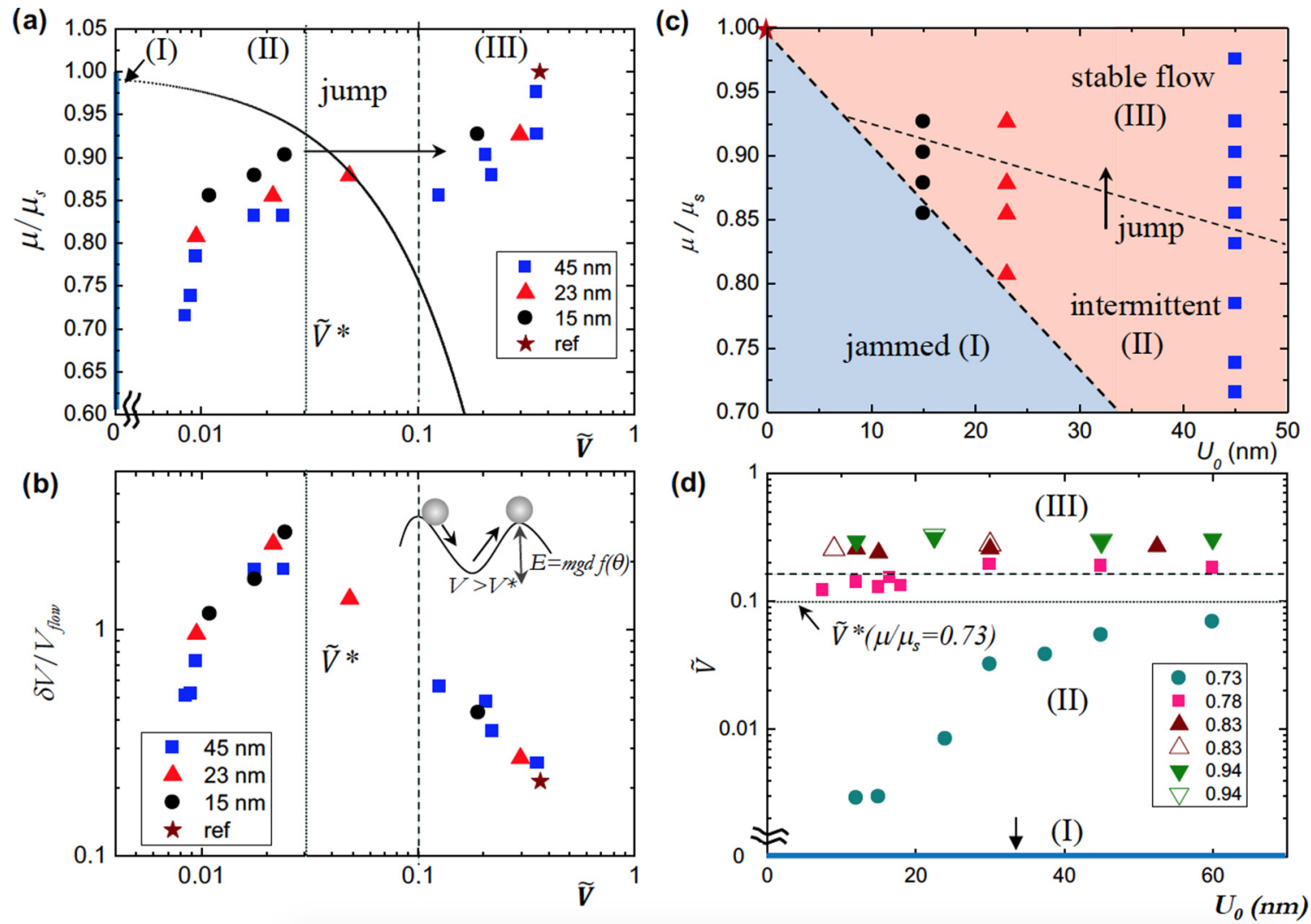

FIG. 3. (a) Normalized friction coefficient $\mu / \mu_{s}$ vs normalized flow velocity $\tilde{V}\left(=V_{\text {flow }} /(g d)^{1 / 2}\right)$ for a layer thickness $h / d=11.6$ (with $d=100 \mu \mathrm{m})$ and $f=40 \mathrm{kHz}$ on the intermediate roughness surface $\left(\theta_{m}=22.5^{\circ}\right)$. The flow is driven by ultrasound amplitudes $U_{0}=15,23$, or $45 \mathrm{~nm}$ (respectively, black circles, red triangles, and blue squares) and the symbol star corresponds to the occurrence of avalanche without ultrasound at the maximum angle $\theta_{m}$. The continuous line corresponds to the model from Ref. [17] (see text). (b) Relative transverse velocity fluctuations vs $\tilde{V}$. Inset illustrates the necessary initial condition $\tilde{V}>\tilde{V}^{*}$ for the grain to escape from a geometric trap formed by its neighbors (see text). (c) Phase diagram constructed by replotting $\mu / \mu_{s}$ from (a) as a function of $U_{0}$ illustrating different states of the system. (d) Variation of $\tilde{V}$ vs ultrasound amplitude $U_{0}(f=40 \mathrm{kHz})$ at various imposed $\mu / \mu_{s}$ for a layer thickness $h_{1} / d=3$ (with $d=500 \mu \mathrm{m}$ ) on the intermediate rough surface $\left(\theta_{m}=20.2^{\circ}\right)$. The open triangles points correspond to a short-burst excitation with 1-s duration. 

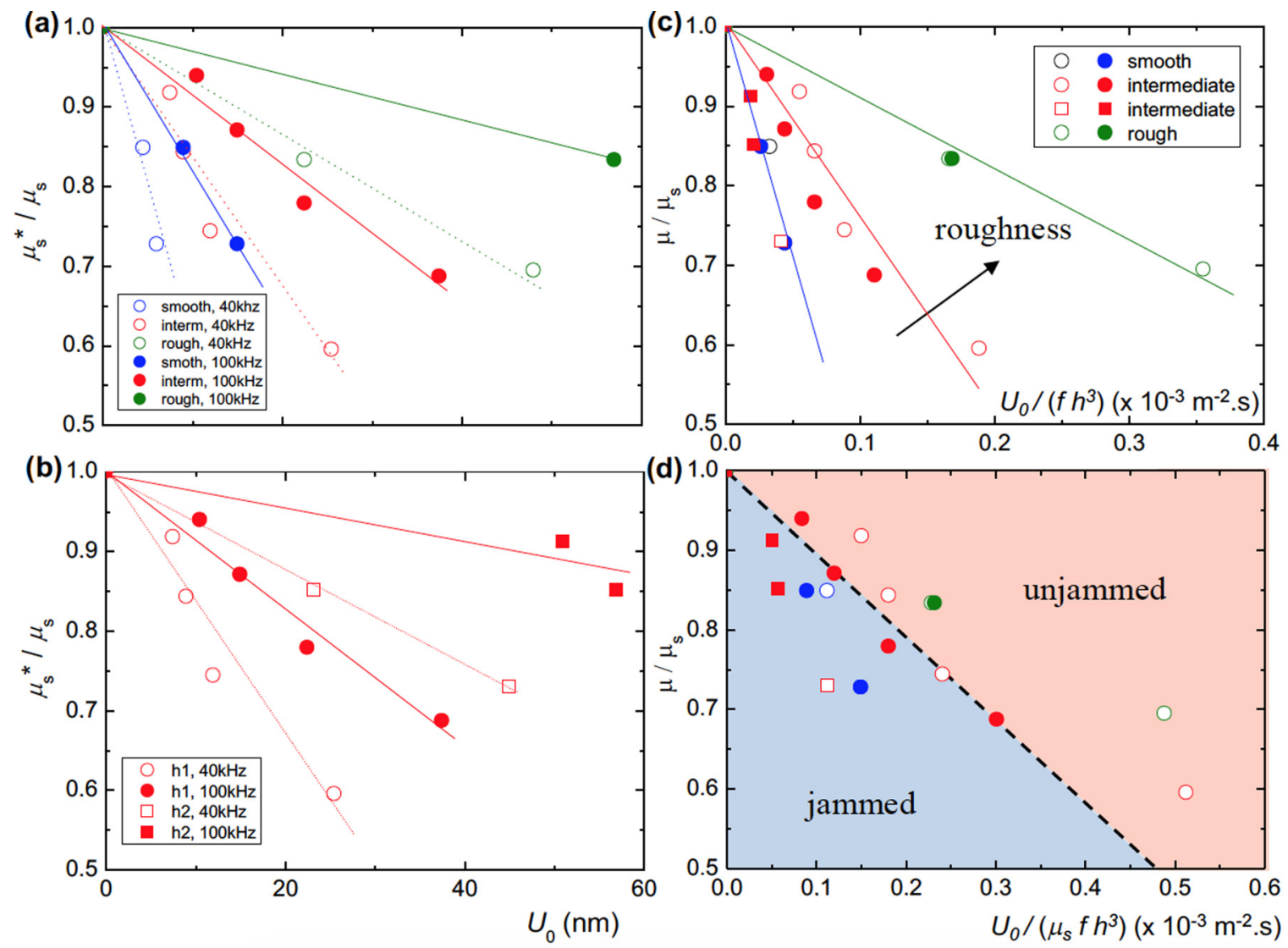

FIG. 4. (a) Variation of the normalized static friction coefficient (threshold) $\mu_{s}^{*} / \mu_{s}$ vs ultrasound amplitude $U_{0}$, for the granular layer with thickness $h_{1} / d=3(d=500 \mu \mathrm{m})$. Blue, red, and green symbols correspond to the smooth, intermediate, and rough surfaces, respectively. Open (filled) symbols correspond to $f=40 \mathrm{kHz}(100 \mathrm{kHz})$. Lines are fits to the data with Eq. (3). (b) Normalized friction coefficient for two layers of different thicknesses, $h_{1} / d=3$ [same symbols as in (a)] and $h_{2} / d=6$ (square points). The surface is of intermediate roughness and the measurements are achieved either at 40 or $100 \mathrm{kHz}$ [same as in (a)]. (c) Data from (a) and (b) plotted against the rescaled amplitude $U_{0} /\left(f h^{3}\right)$, emphasizing the role of surface roughness. (d) All data rescaled by Eq. (3), showing the jammed and unjammed states from either side of the $\mu_{\mathrm{s}}{ }^{*}\left(U_{0}\right)$ line, equivalent to the I-II/III line in Fig 3(c).

flow converges toward the reference state (marked by the star symbols), i.e., the onset of avalanche without ultrasound, in region III [Fig. 3(d)]. There, the inertial flow at a typical velocity $\tilde{V} \gtrsim 1.5 \times 10^{-1}$, used to define region III (dashed line), is mostly insensitive to ultrasound, being only a function of the imposed shear $\mu$ as confirmed, e.g., for $\mu / \mu_{s}=0.83$. We found additionally that in region III, applying the ultrasound either by a short burst (of 1-s duration) or by a continuous wave results in the same flow velocity. All our experiments show that close to $\mu_{s}$, ultrasound vibrations only affect the threshold but not the flow dynamics.

\section{B. Unjamming by ultrasound}

In Fig. 4, we investigate in more details the unjamming by ultrasound of static layers of large beads $(d=500 \mu \mathrm{m})$ at $\mu_{s}{ }^{*}\left(U_{0}\right)<\mu_{s}$ from the jammed state (I). This threshold $\mu_{s}{ }^{*}$ decreases linearly with increasing $U_{0}$ in a systematic manner as for the I-II/III line in Fig. 3(c), which confirms that perturbations by ultrasound excitation and static shear stress play complementary roles in the triggering process. Figures 4(a) and 4(b) show that the unjamming starts at smaller amplitude for low frequency of vibration, low roughness of the transducer, and small thickness of the granular layer. When the unjamming occurs, large flow velocities were found for $\mu$ close to $\mu_{s}$, as those observed in Fig. 3(d).

\section{DISCUSSION}

\section{A. Bifurcation between creeplike flow and self-accelerated inertial flow (macroscopic-scale analysis)}

To understand qualitatively the different regimes of flow triggered by ultrasound (Fig. 3), we use as a guide the heuristic friction model developed by Jaeger et al. [16]:

$$
\mu=\mu_{s} /\left(1+\alpha_{1} \tilde{\gamma}^{2}\right)+\beta \tilde{\gamma}^{2}
$$

Here, $\mu(\dot{\gamma})$ is the normalized shear defined above, $\tilde{\gamma}=$ $\dot{\gamma} \sqrt{d / g_{\perp}}(d$ is the grain size) is the dimensionless flow (shear) rate with $g_{\perp}=g \cos \theta$ and $\dot{\gamma}$ would scale with $V_{\text {flow }} / h$ ( $h$ is related to the sample thickness). $\mu_{s}$ is the granular static friction coefficient, which depends on the grain-grain friction as well as on the depth of the trapping by neighboring grains in the limit $\tilde{\gamma} \rightarrow 0 . \alpha_{1}$ and $\beta$ describe the geometry of the particles and the energy loss during collisions, which involves the coefficient of restitution. Figure 5(a) shows a typical plot of $\mu(\dot{\gamma})$ 
(a)

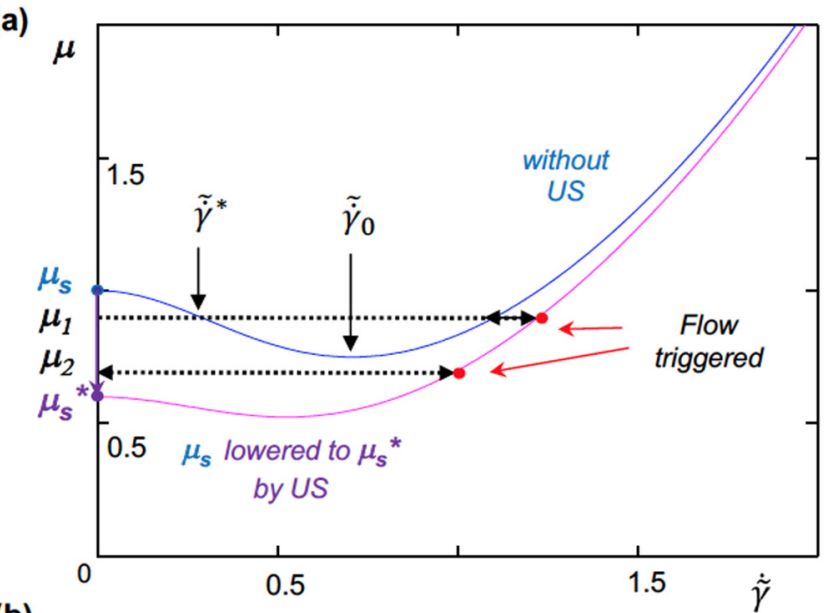

(b)

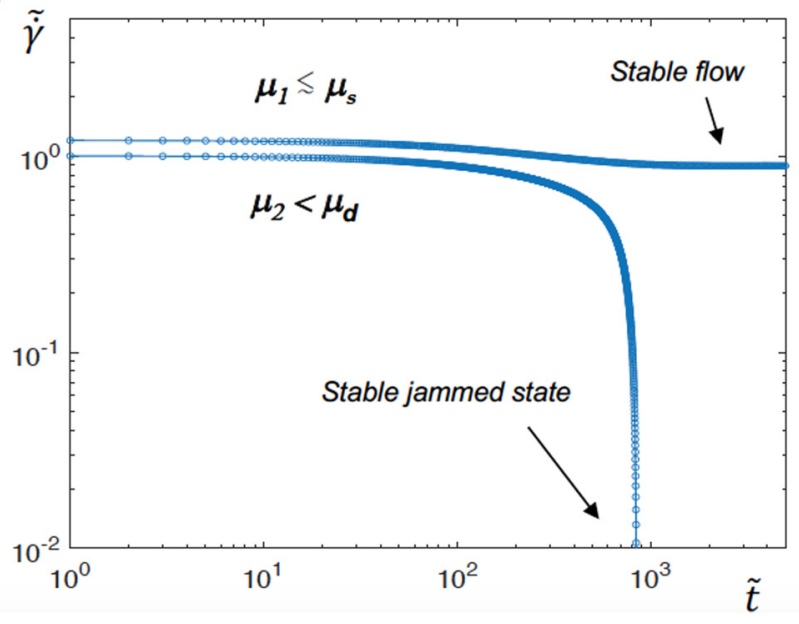

FIG. 5. (a) Effective friction coefficient $\mu$ versus flow rate $\tilde{\gamma}$ derived from Eq. (1) (blue curve) with $\alpha_{1}=2, \beta=0.5$, and $\mu_{s}=1$ as in Ref. [16], which depicts the velocity-weakening behavior for $0<\tilde{\gamma}<\tilde{\gamma}_{0}$. The minimum shear is $\mu_{d}=0.75$ at $\tilde{\gamma}_{0}=0.7$. The application of ultrasound reduces the interparticle friction coefficient, thus lowering the avalanche angle or yield stress from $\mu_{s}=1$ (blue curve) to $\mu_{s}{ }^{*}=0.6$ (pink curve). This triggers the granular flow (red points) as explained in the text. (b) Evolution of the flow rate as a function of time after the removal of ultrasound. The triggered flow bifurcates between a stable inertial flow for imposed shear $\mu_{1}=$ $0.9 \lesssim \mu_{s}$ (with $\tilde{\gamma}_{i}=1.2$ ) and a jamming creep regime for imposed shear $\mu_{2}=0.7<\mu_{d}$ (with $\left.\tilde{\gamma}_{i}=1.0\right)$ (see text).

calculated from Eq. (1) using the parameters as in Ref. [16] where a metastable region is found for $\mu_{d}\left(\tilde{\gamma}_{0}\right)<\mu<\mu_{s}$ corresponding to the negative slope for $0<\tilde{\gamma}<\tilde{\gamma}_{0}$, as shown by Fig. 1(d). In such a case, as proposed there, the unlimited flow is possible only when the initial flow rate or velocity is larger than $\dot{\gamma}^{*}$ or $V^{*}$ (see also discussion below) given by the velocity-weakening part of $\mu(\dot{\gamma})$ as we likely observed in the jump region of Fig. 3(a). This velocity-weakening regime can initiate the stick-slip frequently observed in solid friction and granular media, due to aging-rejuvenation competition acting at contact asperities $[1,20]$. In solid friction, this leads to the bifurcation between creep motion and self-accelerated slip at a multicontact interface via an increasing oscillating shear force close to the yielding [28]. In granular media, we may expect a similar aging-rejuvenation competition in which contact asperities are replaced by grain contacts [12]. Depending on the imposed shear $\mu$ relative to the static threshold $\mu_{s}$ and the initial shear rate $\tilde{\gamma}$, the granular dynamics could bifurcate between a self-accelerated flow (avalanche in region III) and a creep which stops to flow at long time range and recovers the static state (in region I) [16,17,25].

Let us now consider the effect of ultrasound for these two different flows in granular layers. As reported in Ref. [12], the ultrasonic vibration can induce the frictional slip between grain contacts, leading to the frictional dissipation, the softening of the shear contact stiffness, and accordingly of the macroscopic modulus in a granular medium. Moreover, we have shown in the configuration of a single sphere-plane contact that such oscillation-induced frictional slip can also lubricate acoustically the contact, leading to the reduction of the static threshold from $\mu_{s}$ to $\mu_{s}{ }^{*}$ [42] (see discussion below). Such a decrease of the threshold modifies the above friction model as shown in Fig. 5(a) where the reference blue curve, corresponding to $\mu_{s}$, is lowered to the pink one with $\mu_{s}{ }^{*}<\mu_{s}$. The avalanche is then triggered by ultrasound whenever the granular is loaded such that $\mu>\mu_{s}{ }^{*}$-the main observation of this work. More specially, we propose the scenario as follows for two different imposed shear: close to the threshold such that $\mu=\mu_{1}$ with $\mu_{d}\left(\tilde{\gamma}_{0}\right)<\mu_{1} \lesssim \mu_{s}$, and far below the threshold with $\mu=\mu_{2}<\mu_{d}$. For $\mu_{1} \lesssim \mu_{s}$, the flow is triggered from the metastable state and persists when the ultrasound is turned off since the $\tau-\dot{\gamma}$ relation recovers to the initial curve (blue). Indeed, the system is now driven to the inertial flow region (III), dominated by the term $\beta \tilde{\gamma}^{2}$ in Eq. (1), where the static coefficient of friction $\mu_{s}$ and hence the ultrasound-induced decrease $\mu_{s}{ }^{*}$ are expected to have a vanishing effect. This corresponds precisely to our experimental observations in Fig. 3(d) for $\mu_{1}>0.76$, where the data obtained with either the short burst or the continuous ultrasonic excitation are indistinguishable. By contrast, for imposed shear $\mu_{2}<\mu_{d}$ (minimum value in the blue curve) the granular system stops to flow in the absence of ultrasound and goes back to the initial solution at $\tilde{\gamma}=0$, i.e., the stable jammed state (I). Moreover, in the latter case, the velocity of triggered flow increases with increasing ultrasound amplitude by lowering further $\mu_{s}$ [42], as for the experimental observation in Fig. 3(d) with $\mu=0.73$.

We may also investigate this bifurcation phenomenon from the given initial condition to the stable states as a function of time $[28,46]$. To do so, we consider the motion of a single mass ("slider") on an inclined plane [inset of Fig. 1(b)] within the framework of a mean-field approximation. It is submitted to a shear force by gravity and a friction by the phenomenological friction law in Eq. (1). The mean acceleration approximately written as $d\langle V\rangle / d t$ with $\langle V\rangle \sim V_{\text {flow }}$ (denoted as $V$ below for simplicity) is given by $[16,17]$ : $m(d V / d t)=F_{t}-\mu F_{n}$ where the shear force $F_{t}=m g \sin \theta$ is imposed by the inclined plane, $F_{n}=m g \cos \theta$ and the friction coefficient $\mu$ given by Eq. (1). Using the dimensionless unit $\tilde{t}=t / \sqrt{d / g_{\perp}}$, we write the equation of motion as

$$
d \tilde{\gamma} / d \tilde{t}=\tan \theta-\mu_{s} /\left(1+\alpha_{1} \tilde{\gamma}^{2}\right)-\beta \tilde{\gamma}^{2} .
$$

Figure 5(b) depicts the solutions of the flow velocity as a function of time, calculated from Eq. (2) with the initial 
flow rates $\tilde{\gamma}_{i}$ corresponding to the triggered flows under two imposed shears [red circles in Fig. 5(a)], $\mu_{1}=\tan \theta_{1}$ with $\tilde{\gamma}_{i}=1.2$ and $\mu_{2}=\tan \theta_{2}$ with $\tilde{\gamma}_{i}=1$, respectively. For the inclined plane at $\theta_{1}$, the system reaches a steady inertial flow at long time whereas for the lower inclination $\theta_{2}$, the flow slows down and arrests quickly, being consistent with the above analysis via Fig. 5(a). Qualitatively, such a bifurcation agrees well with our experiments on triggered granular flows [Figs. 3(c) and 3(d)]. It is worth noting that in yield stress fluids such as foams, emulsions, and polymers the thixotropic effect resulting from the competition between structuration and destruction by shear can also lead to a viscosity bifurcation as a function of time [46].

\section{B. From creep to inertial granular flows (local-scale analysis)}

Simulations and experiments [47] showed that in the quasistatic regime at a very low imposed flow velocity, external driving induces large velocity fluctuations of grains when the system is far below the yield stress, associated with the complex dynamics of a grain confined by high local static threshold. However, at high flow velocity, the relative velocity fluctuations decrease with the flow velocity [48]. Here, Fig. 3(b) shows that all data collapse on the same curve when plotting the relative transverse velocity fluctuations $\delta V / V_{\text {flow }}$ versus flow velocity, independent of the ultrasound amplitude and of the inclination.

Around a threshold flowing velocity $\tilde{V}^{*} \sim 0.03$, the relative velocity fluctuations $\delta V / V_{\text {flow }}$ abruptly becomes a decreasing function of $V_{\text {flow }}$ (or $\tilde{V}$ ) similar to previous studies $[47,48]$. Above $\tilde{V}^{*}$, the observed small relative velocity fluctuations are characteristic of the large failure event dominated by the inertial number: the granular layer is in the inertial flow state (region III), corresponding to highly uncorrelated grain motion [49]. There, the dynamics arising from the naturally occurring avalanche at $\mu_{s}$ and the triggered flows are indistinguishable [Fig. 3(b)], which is consistent with the observation in Fig. 3(a) where the effect of ultrasound is negligible during the fast continuous flow. This is also in line with the results in Fig. 3(d) close to $\mu_{s}$ where triggered avalanches flow at the same velocity with or without prolonged ultrasound.

Below $\tilde{V}^{*}$, for $\mu / \mu_{\mathrm{s}} \ll 1$, perturbations such as shear and vibration can induce some grain motion but the momentum transfer is insufficient for a global flow (avalanche), given the low static load. In the intermittent regime for an imposed shear load near the metastable zone, the averaged creep flow induced by ultrasound increases with the vibration amplitude, as shown in Fig. 3(d) for $\mu / \mu_{s}=0.73$. This is probably due to the decrease of the interparticle friction coefficient $\mu_{g}$ induced by the acoustic lubrication which ensures a decreased frictional loss during local slipping events.

The robust superposition of the relative velocity fluctuations in Fig. 3(b) suggests that the order of magnitude of the threshold velocity $V^{*}$ is given by the intrinsic dynamics of the granular system. The intensity of the threshold velocity results from the kinetic energy acquired by the grains during the ultrasound-induced rearrangement events, and should be large enough for the grains not to remain trapped after collisions. Then, one needs $(1 / 2) m V^{* 2}=E(\theta)=\operatorname{mgd} f(\theta)$ if the shocks are elastic [see inset of Fig. 3(b)] with $f(\theta) \sim[1-$ $\left.\cos \left(\theta-\theta_{m}\right)\right] \sim\left(\theta-\theta_{m}\right)^{2} / 2$, corresponding to a geometric parameter [17]. This gives a minimum velocity $V(\mu) \geqslant V^{*}=$ $\left(\mu_{s}-\mu\right)(g d)^{1 / 2}$ for escaping the potential energy trap near the threshold. As shown Fig. 3(a) this order of magnitude for the threshold velocity $\tilde{V}^{*} \sim \mu_{s}-\mu \sim 0.04$ (with $\mu_{s}=$ $\left.\tan \theta_{m} \approx 0.41\right)$ corresponds consistently to the location of the velocity jump $\tilde{V}^{*}(\sim 0.03)$ observed in the curves $\mu(\tilde{V})$ at imposed shear around $\mu / \mu_{s} \sim 0.9$ and define a transitional boundary separating conveniently region II, where the flow velocity depends on the amplitude, from the inertial flow region III. We may also estimate the characteristic velocity for the system with larger beads in Fig. 3(d) at $\mu / \mu_{s}=0.73$ (region II), $\tilde{V}^{*} \sim 10^{-1}$ (with $\mu_{s} \approx 0.37$ ). Again, the separation between the different dynamic behaviors II/III matches the experiment. The results are summarized schematically in Fig. 5(a) where $\dot{\tilde{\gamma}}^{*}(\sim 0.3)$ is obtained from the intersection $\mu_{1}$ with the curve $\mu(\dot{\gamma})$ in the unstable region.

\section{Rearrangement of grains unjammed by ultrasound}

As mentioned above, in a confined granular material, ultrasound can weaken the shear contact stiffness via microslip at grain contacts, inducing the frictional dissipation and reducing the material rigidity $[12,13,50]$. For a single grain under gravity [42], such shear ultrasound-induced decrease of the stuck area of a (hertzian) contact results in sliding below the static threshold. We surmise that a similar mechanism is at work here for the granular layer on the macroscopic scale.

As indicated above in Eq. (1), the static friction coefficient $\mu_{s}=\mu_{p}+\mu_{g}\left(=\tan \theta_{m}\right)$ includes both the interparticle friction $\mu_{p}$ and the geometric trapping $\mu_{g}$ (dilatancy effect). Because of the small amplitude of ultrasound, the soundmatter interaction only modifies $\mu_{p}$ but not $\mu_{g}$, hence $\mu_{s}{ }^{*}=$ $\mu_{p}{ }^{*}+\mu_{g}$. From the Mindlin friction model, we have shown that decreases of both the shear contact stiffness $\Delta k_{t} / k_{t}$ [12] and the interparticle friction coefficient $\Delta \mu_{p} / \mu_{p}$ [42] are approximately proportional to $\sim-f_{t} /\left(\mu_{p} f_{n}\right)$, where $f_{t}$ is the oscillating tangential force and $f_{n}$ the static normal force at a single contact. Such a scaling of vibration-induced softening $\sim-F_{a c} /\left(\mu_{p} W\right)$ can also be generalized to the effective shear contact stiffness at a multicontact interface between solids [51] and to the shear modulus [13] and the yield stress [52] in a vibrated granular medium where polydisperse contact asperities are replaced by grain contacts and inertial effects are negligible. Here $F_{a c}$ is the imposed macroscopic oscillating shear force, $W$ the normal load, and $\mu$ the (averaged) effective interparticle friction coefficient. Accordingly, we adopt here this scaling formula for the granular layer to describe the reduction of $\Delta \mu_{p} / \mu_{p} \sim-F_{a c} /\left(\mu_{p} W\right)$, which implies that $\mu_{s}^{*} / \mu_{s} \sim 1-F_{a c} /\left(\mu_{s} W\right)$.

For the granular layer considered here [Fig. 1(b)], we may write the above shear oscillating force by $F_{a c}=\hat{k} \hat{U}$ with $\hat{k}$ the effective stiffness of the granular layer and $\hat{U}$ the averaged displacement amplitude of shear vibration over the thickness $h(\approx 1.5 \mathrm{~mm})$. To evaluate $\hat{U}$, we assume the ultrasound amplitude at a distance $z$ from the transducer to be given by $U(z) \sim U_{0} \exp (-z / \delta)$ with $U_{0}$ the source amplitude and $\delta(\sim \lambda=c / f)$ the attenuation length dominated by wave scattering [7]. Thus we have $\hat{U}=(1 / h) \int_{0}^{h} U_{0} \exp (-z / \delta) d z=$ $(\delta / h) U_{0}[1-\exp (-h / \delta)]$. With a wave speed $c \sim 10 \mathrm{~m} / \mathrm{s}$ 
$[13,50]$ and ultrasound frequency $f=70 \mathrm{kHz}$, the wavelength is $\lambda \sim 0.15 \mathrm{~mm}$, leading to $\delta$ much less than $h$ and consequently $\hat{U} \sim(\delta / h) U_{0} \sim(c / f h) U_{0}$. Using an estimate for $W \sim(\rho g h) L^{2}$ with $L$ the width of the ultrasonic transducer [Fig. 1(b)] and $\rho$ the density of medium, and the relation between the effective stiffness and shear modulus of the layer $\hat{k} \sim G\left(L^{2} / h\right)$ as well as the sound speed $c=(G / \rho)^{1 / 2}$, we obtain the following expression:

$$
\mu_{s}{ }^{*} / \mu_{s} \sim 1-\left(c^{3} / g\right)\left[U_{0} /\left(\mu_{s} f h^{3}\right)\right] .
$$

Equation (3) contains the sufficient dependencies for rescaling the experimental data for various layer thicknesses and ultrasound amplitudes and frequencies [Fig. 4(c)]. The roughness of the transducer surface shall pin a small fraction of the grains in the vicinity of the solid surface and increase the effect of local dilatancy by the parameter $\mu_{g}$, therefore decreasing the slope in Eq. (3), provided that $\mu_{p}$ is similar for all the systems studied. This predicted scaling agrees with the observation in Fig. 4(c) in which rougher surface (higher $\left.\mu_{s}\right)$ requires larger vibration amplitude $\left(U_{0}\right)$ to provoke the grain motion from jammed to unjammed states. Moreover, by fitting the mean slope of the rescaled plot in Fig. 4(d) (black dashed line), we may estimate from Eq. (3) the wave speed $c=20 \pm 5(\mathrm{~m} / \mathrm{s})$, which agrees well with those found in the previous works $[13,50]$. Nevertheless, the points remain scattered after rescaling in Fig. 4(d) $(\Delta c / c \sim 25 \%)$. More comprehensive studies are required in future investigation to quantify the effects of the layer thickness and ultrasound frequency for a better understanding of the unjamming transition between region I and region II/III [Fig. 1(c)].

Since we did not detect any effect of the polarization of the ultrasounds, the phenomenology observed here is similar to Ref. [42], where ultrasound was shown to play the role of an effective temperature in the unjamming diagram. The analysis shows that the static threshold of granular layers is lowered by ultrasound via the decrease of the interparticle friction coefficient, resulting in the remarkable alteration of the dynamics at the macroscale with the triggering of granular avalanches.

\section{CONCLUSION}

In summary, we have investigated the flow of granular layers triggered by nanometer-amplitude ultrasound below the threshold. When the angle of inclination is far below the angle of repose $\theta_{r}$, the ultrasound induces highly fluctuating grain motions with a small average flow velocity. As opposed to shaking experiments, grain motions do not correspond to geometrical rearrangements such as jumps forced by the oscillations, but rather to jigglings or agitations in traps formed by neighbors thanks to the small potential energy released from the decrease of interparticle friction $\mu_{p}$ by the shear acoustic lubrication at grain contacts. This sound-matter interaction depends on ultrasound frequency and layer thickness due to wave attenuation, as well as on the transducer surface roughness which pins the grains by the dilatancy effect.

Close below the angle of repose, we observe the triggered flow with a larger averaged velocity increasing with the ultrasound amplitude, owing to the reduction of the granular threshold $\mu_{s}$ via the decrease of interparticle friction $\mu_{p}$. This creeplike flow can be sustained only by a prolonged excitation and arrests when ultrasound is turned off. At higher inclination, close to $\theta_{m}$, triggered avalanches are fast and continuous with a flow velocity independent of the applied ultrasound; it is dominated by the inertial self-generated dynamics of the granular system. These experimental observations agree with the predictions of any velocity-weakening friction model, consistent with the picture of the unjamming transition.

We believe that this work provides a unified picture of the behavior of vibrated granular matter. The multiscale analysis will be useful to better understand the local and remote dynamic triggering of landslides and earthquakes by seismic waves, including aftershocks [2,21].

\section{ACKNOWLEDGMENTS}

We thank T. Baumberger, C. Caroli, P. Johnson, H. Bhat, and P. Bernard for helpful discussions. This work was supported by French LABEX WIFI under References No. ANR-10-LABX-24 and No. ANR-10-IDEX-0001-02, and by ERC-CG-2013-PE10-617472 SLIDEQUAKES.
[1] C. Marone, Annu. Rev. Earth Planet. Sci. 26, 643 (1998).

[2] C. Scholz, The Mechanics of Earthquakes and Faulting, 3rd ed. (Cambridge University Press, Cambridge, UK, 2019).

[3] J. Gomberg, P. A. Reasenberg, P. Bodin, and R. A. Harris, Nature (London) 411, 462 (2001).

[4] R. Bürgmann, Nature (London) 512, 258 (2014).

[5] V. Durand, A. Mangeney, F. Hass, X. Jia, and A. Peltier et al., J. Geophys. Res. 123, 2422 (2019).

[6] C.-H. Liu and S. R. Nagel, Phys. Rev. Lett. 68, 2301 (1992).

[7] X. Jia, C. Caroli, and B. Velicky, Phys. Rev. Lett. 82, 1863 (1999).

[8] H. A. Makse, N. Gland, D. L. Johnson, and L. Schwartz, Phys. Rev. E 70, 061302 (2004).

[9] E. T. Owens and K. E. Daniels, Europhys. Lett. 94, 54005 (2011).
[10] P. Johnson and X. Jia, Nature (London) 437, 871 (2005).

[11] P. Johnson, H. Savage, M. Knuth, J. Gomberg, and C. Marone, Nature (London) 451, 57 (2008).

[12] X. Jia, T. Brunet, and J. Laurent, Phys. Rev. E 84, 020301(R) (2011).

[13] J. Brum, J.-L. Gennisson, M. Fink, A. Tourin, and X. Jia, Phys. Rev. E 99, 042902 (2019).

[14] J. Y. Yoritomo and R. L. Weaver, Phys. Rev. E 102, 012901 (2020).

[15] A. J. Liu and S. R. Nagel, Nature (London) 396, 21 (1998).

[16] H. M. Jaeger, C. Liu, S. R. Nagel, and T. A. Witten, Europhys. Lett. 11, 619 (1990).

[17] L. Quartier, B. Andreotti, S. Douady, and A. Daerr, Phys. Rev. E. 62, 8299 (2000). 
[18] A. Baldassarri, F. Dalton, A. Petri, S. Zapperi, G. Pontuale, and L. Pietronero, Phys. Rev. Lett. 96, 118002 (2006).

[19] J. A. Dijksman, G. H. Wortel, L. T. H. van Dellen, O. Dauchot, and M. van Hecke, Phys. Rev. Lett. 107, 108303 (2011).

[20] T. Baumberger and C. Caroli, Adv. Phys. 55, 279 (2006).

[21] R. Burridge and L. Knopoff, Bull. Seismol. Soc. Am. 57, 341 (1967).

[22] S. Brown, C. Scholz, and J. Rundle, Geophys. Res. Lett. 18, 215 (1991).

[23] O. Pouliquen and N. Renaut, J. Phys. II France 6, 923 (1996).

[24] A. Daerr and S. Douady, Nature (London) 399, 241 (2000).

[25] P. Coussot, Q. D. Nguyen, H. T. Huynh, and D. Bonn, Phys. Rev. Lett. 88, 175501 (2002).

[26] M. Wyart, Europhys. Lett. 85, 24003 (2009).

[27] S. Nasuno, A. Kudrolli, and J. P. Gollub, Phys. Rev. Lett. 79, 949 (1997).

[28] L. Bureau, T. Baumberger, and C. Caroli, Phys. Rev. E 64, 031502 (2001).

[29] E. J. R. Parteli, M. A. F. Gomes, and V. Brito, Phys. Rev. E 71, 036137 (2005).

[30] B. N. J. Persson, Sliding Friction, Nanoscience and Technology Series (Springer, Heidelberg, 1998).

[31] H. M. Jaeger, C.-H. Liu, and S. R. Nagel, Phys. Rev. Lett 62, 40 (1989).

[32] E. Clement and J. Rajchenbach, Europhys. Lett. 16, 133 (1991).

[33] G. D'Anna, P. Mayor, A. Barrat, V. Loreto, and F. Nori, Nature (London) 424, 909 (2003).

[34] V. Zaloj, M. Urbakh, and J. Klafter, Phys. Rev. Lett. 82, 4823 (1999).

[35] A. Cochard, L. Bureau, and T. Baumberger, J. Appl. Mech. 70, 220 (2003).
[36] H. Melosh, Nature (London) 379, 601 (1996).

[37] M. O'Reilly and S. Brown, Cyclic Loading of Soils: From Theory to Experiments (Blackie and Son Ltd., Glasgow, UK, 1991).

[38] G. Metcalfe, S. G. K. Tennakoon, L. Kondic, D. G. Schaeffer, and R. P. Behringer, Phys. Rev. E 65, 031302 (2002).

[39] S. Aumaitre, C. Puls, J. N. McElwaine, and J. P. Gollub, Phys. Rev. E 75, 061307 (2007).

[40] C. Hanotin, S. Kiesgen de Richter, P. Marchal, L. J. Michot, and C. Baravian, Phys. Rev. Lett. 108, 198301 (2012).

[41] H. Lastakowski, J. Geminard, and V. Vidal, Sci. Rep. 5, 13455 (2015).

[42] J. Léopoldes, G. Conrad, and X. Jia, Phys. Rev. Lett 110, 248301 (2013)

[43] See Supplemental Material at http://link.aps.org/supplemental/ 10.1103/PhysRevE.102.042901 for movies of avalanches.

[44] http://www.rowland.harvard.edu/labs/bacteria/software/index. php.

[45] F. Radjai and S. Roux, in Nonsmooth Mechanics and Analysis, edited by P. Alart, O. Maisonneuve, and R. Rockafellar (Springer, New York, 2006), Chap. 20, pp. 233-245.

[46] F. Da Cruz, F. Chevoir, D. Bonn, and P. Coussot, Phys. Rev. E 66, 051305 (2002).

[47] GDR MiDi, Eur. Phys. J. E 14, 341 (2004)..

[48] F. da Cruz, S. Emam, M. Prochnow, J. N. Roux, and F. Chevoir, Phys. Rev. E. 72, 021309 (2005).

[49] O. Pouliquen, Phys. Rev. Lett. 93, 248001 (2004).

[50] S. van den Wildenberg, M. van Hecke, and X. Jia, Europhys. Lett. 101, 14004 (2013).

[51] L. Bureau, C. Caroli, and T. Baumberger, Proc. R. Soc. London A 459, 2787 (2003).

[52] S. Wildenberg, X. Jia, J. Léopoldès, and A. Tourin, Sci. Rep. 9 , 5460 (2019). 